\title{
Super-Resolution-Based DOA Estimation with Wide Array Distance and Extrapolation for Vital FMCW Radar
}

\author{
Sangdong $\operatorname{Kim}^{1,2} \cdot$ Bongseok Kim ${ }^{1} \cdot$ Youngseok Jin ${ }^{1} \cdot J^{\prime}$ onghun Lee ${ }^{1,2, *}$
}

\begin{abstract}
This paper proposes a super-resolution-based direction-of-arrivals (DOA) estimation with wide array distance and extrapolation for vital frequency-modulated continuous-wave (FMCW) radar. Most super-resolution algorithms employ the distance between adjacent arrays of half a wavelength, i.e., $\lambda / 2$. Meanwhile, in the case of narrow field of view of FMCW radar, the resolution of the angle is maintained by increasing the spacing between the arrays even if the number of arrays decreases. In order to employ these characteristics of array spacing and resolution, the proposed algorithm confirms whether or not to use the case where the distance between the adjacent arrays is greater than $\lambda / 2$. In the case of an array distance $>\lambda / 2$, a super-resolution algorithm is performed to obtain the enhanced DOA resolution. Moreover, the proposed algorithm virtually generates data between antennae by using extrapolation in order to further improve the performance of the resolution. The simulation results show that the proposed algorithm achieves the results of root-mean-square error similar to conventional super-resolution algorithms while maintaining low complexity. In order to further verify the performance of the proposed estimation algorithm, we demonstrate its employment in practice: experiments in a chamber room and an indoor room were conducted.
\end{abstract}

Key Words: Array Distance, DBF, DOA, Super-Resolution, Vital FMCW Radar.

\section{INTRODUCTION}

Due to the current advancements in technologies, such as self-driving vehicles and drones, a growing need for improvements in cameras and radar sensors to detect targets has presented. Unlike other sensors like the camera and LIDAR (light detection and ranging) [1], radar sensors can detect targets reliably because they are insensitive to inclement weather, such as fog and heavy rain. Because of these advantages, a variety of radar sensors have been utilized for several applications, including self-driving vehicles, drones, and aircraft [2]. Importantly, radar sensors are also deployed in military devices, such as for the detection of enemy persons, and in surveillance devices [3,
4]. To detect humans, radar can detect the vital signs of people in surveillance and rescue operations. Among the vital radar sensors, frequency-modulated continuous-wave (FMCW) radar has been widely used due to its simplicity.

In order to obtain multiple parameters, such as the distance, vital Doppler, or angle, low complexity fast Fourier transform (FFT)-based algorithms have been used in existing FMCW radar. Especially, for angle detection, the direction-of-arrivals (DOA) estimation of humans is key to finding a precise position for vital FMCW radar $[5,6]$. To this end, digital beamforming (DBF) as an FFT-based DOA estimation method has been utilized. However, the FFT-based parameter estimator has low resolution and accuracy [7]. Because the resolution of the

Manuscript received March 9, 2020 ; Revised June 12, 2020 ; Accepted September 3, 2020. (ID No. 20200309-029J)

${ }^{1}$ Division of Automotive Technology, Daegu Gyeongbuk Institute of Science \& Technology, Daegu, Korea.

${ }^{2}$ Department of Interdisciplinary Engineering, Daegu Gyeongbuk Institute of Science \& Technology, Daegu, Korea

"Corresponding Author: Jonghun Lee (e-mail: jhlee@dgist.ac.kr)

This is an Open-Access article distributed under the terms of the Creative Commons Attribution Non-Commercial License (http://creativecommons.org/licenses/by-nc/4.0) which permits unrestricted non-commercial use, distribution, and reproduction in any medium, provided the original work is properly cited.

(c) Copyright The Korean Institute of Electromagnetic Engineering and Science. All Rights Reserved. 
FFT is low, the DOA estimation exploits super-resolution algorithms, for instance, the estimation of signal parameters via rotational invariance techniques (ESPRIT) and multiple signal classification (MUSIC).

Notably, a super-resolution algorithm with better resolution was proposed. In [8], the method first finds the autoregressive (AR) parameters via an initial DOA result and then uses these AR parameters for linear extrapolation. For fine DOA estimations, extrapolated signals are fed to the input of the ESPRIT algorithm. In [9], a super-resolution algorithm is applied in low signal-to-noise ratio (SNR) environments. This algorithm uses a partition window to obtain the super-resolution results.

However, the algorithms listed above still have limited resolution. To improve the resolution of the DOA, this paper focuses on the change in the array element distance. The distance between the adjacent antenna array elements is usually set to $\lambda / 2$ because the optimal range of the field of view (FOV) is $\pm 90^{\circ}$. However, this paper focuses on a narrow FOV since conventional studies of vital radar considers a narrow FOV [5]. In [10], $\mathrm{K}$-band portable FMCW radar with beamforming is represented. That radar system has a four-element linear beamforming array with the distance between adjacent elements $=\lambda / 2$. When the distance between the adjacent elements is $\lambda / 2$, the FOV is set to $\pm 90^{\circ}$. However, in [10], effective angle detection is performed within $\pm 30^{\circ}$. Therefore, the FOV of [10] needs to be set at $\pm 30^{\circ}$ when the distance between adjacent elements is $\lambda$. Further, [11] represents phased-array Doppler radar using MIMO beamforming techniques. [11] is also set with the distance between adjacent elements equal to $\lambda / 2$. [11] focuses on interesting targets within $\pm 30^{\circ}$ of the FOV. Therefore, [11] also needs to set a narrow FOV for interesting targets.

In narrow FOVs, a margin to increase the spacing between arrays exists. The resolution of the angle estimation is maintained by increasing the spacing between arrays even if the number of arrays decreases. By reducing the total number of arrays, the proposed scheme reduces the system complexity while still maintaining the same resolution. Then, using extrapolation, the proposed algorithm with a wide array distance virtually generates data between the antenna spacing to improve resolution performance. Finally, in almost all super-resolution algorithms, including ESPRIT and MUSIC, the distance between adjacent arrays is $0.5 \lambda$. To enhance the resolution of the super-resolution DOA algorithm, the distance between antennae may be changed. Especially when the FOV of interest for vital radar is under $\pm 30^{\circ}$, the array distance must be set to $\lambda$ instead of $\lambda / 2[12]$.

Taking these limitations into account, this paper proposes a super-resolution-based DOA estimation for vital FMCW radar with a wide array distance. Initially, we set the FOV of interest in the specific radar application. For instance, we set the FOV to be $\pm 30^{\circ}$. In accordance with this FOV, the array distance is set to $\lambda$. Next, when the super-resolution-based DOA algorithm is executed, we obtain improved resolution results compared to those from conventional radar.

This paper is arranged as follows. Section II briefly reviews the signal model for the distance, the vital Doppler, and the angle of the vital FMCW radar. Section III proposes the superresolution DOA estimator with a wide array distance for the vital FMCW radar. This section also presents an analysis of the complexity of each algorithm. Sections IV and V represent the simulations and experimental results for the various environments, respectively. Finally, conclusions are shown in Section VI.

\section{SIGNAL MODEL}

The system models of the vital FMCW radar is shown in this section. Vital FMCW radar is ultimately responsible for the estimation of multiple parameters of the human being. Chiefly, the respiration and heartbeat Doppler signals are interesting parameters from the reflected FMCW signal. An FMCW transmitted (TX) signal is generated from the radar system. The reflected signal from multiple human beings is obtained at the receiver (RX). As a beat signal, the reflected signal is changed into a sinusoidal signal [5]. The sinusoidal signal's frequency is proportional to the time delay between the FMCW radar and the human being. The system model of the FMCW radar is considered for multiple human beings. Multiple RX antenna arrays and a TX antenna are examined for suitability.

The vital FMCW TX signal is represented in Fig. 1 whereby in

$$
s_{\mathrm{tx}}(t)=\sum_{l=0}^{L-1} s_{0}\left(t-l T_{F}\right),
$$

$L$ represents the number of FMCW chirp signals, $T_{F}$ defines the total duration of the chirp symbol (e.g., $T_{F}=T+T_{i}$ ), $T$ is the chirp symbol's duration, and $T_{i}$ is the guard period's duration. The chirp symbol for vital FMCW radar $s_{0}(t)$ is composed of

$$
s_{0}(t)=\left\{\begin{array}{cc}
\exp \left(j\left(2 \pi f_{0} t+\frac{\mu t^{2}}{2}\right)\right), & \text { for } 0 \leq t<T \text { and }, \\
0, & \text { elsewhere }
\end{array}\right.
$$

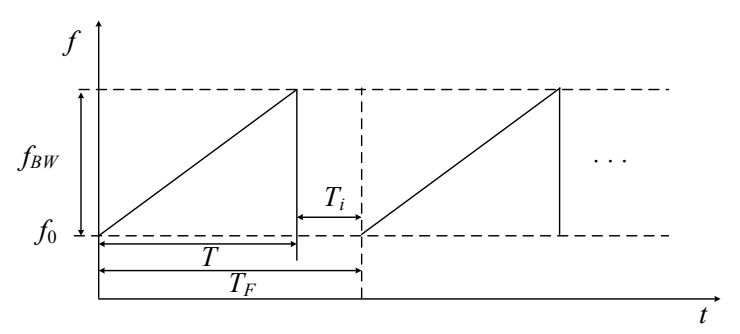

Fig. 1. Transmitted signal of the FMCW radar. 
where $f_{0}$ is the start frequency of the bandwidth, $\mu=2 \pi f_{B W} / T$ is the FMCW chirp symbol's frequency slope as time progresses, and $f_{B W}$ is the FMCW signal's bandwidth.

In order to estimate vital Doppler information of $M$ targets, the definition of body movement is needed to determine the target's fixed distance $d_{0, m}$ and the time-varying distance $x_{m}(t)$ of the $m$-th target, such as chest displacements caused by respiration and the heartbeat. The time-varying distance between the radar and the human is represented by

$$
d_{m}(t)=d_{0, m}+x_{m}(t),
$$

where $x_{m}(t)=x_{m, \mathrm{~h}}(t)+x_{m, \mathrm{r}}(t)$, and $x_{m, \mathrm{r}}(t)$ and $x_{m \mathrm{~h}} \mathrm{~h}(t)$ define the $m$-th human's body motions by respiration and heartbeat, respectively. The time-varying distance movement $x_{m}(t)$ is composed of

$$
x_{m}(t)=a_{m, \mathrm{~h}} \sin \left(2 \pi f_{m, \mathrm{~h}} t\right)+a_{m, \mathrm{r}} \sin \left(2 \pi f_{m, \mathrm{r}} t\right),
$$

In the case of a uniform linear array (ULA) of $K$ antennae, the $m$-th human's received signal is obtained with a time delay $\tau_{m}$. The received signal $y_{k,}(t)$ in the $l$-th chirp symbol and the $k^{-}$ th antenna array is represented [5] in (5), where $a_{m, \mathrm{~h}}$ and $a_{m, \mathrm{r}}$ define the amplitude of the heartbeat and respiration, respectively, and $f_{m, \mathrm{~h}}$ and $f_{m, \mathrm{r}}$ denote the frequency of the heartbeat and respiration, respectively.

$$
\begin{aligned}
y_{k, l}(t)= & \sum_{m=0}^{M-1}\left[\tilde{a}_{m} s_{\mathrm{tx}}\left(t-\tau_{m}\right) \exp \left(\frac{j 4 \pi l T_{F}}{\lambda}\left(x_{m, \mathrm{~h}}(t)+\sum_{p=1}^{P} x_{m, \mathrm{r}, p}(t)+\xi\right)\right) .\right. \\
& \left.\exp \left(\frac{j 2 \pi}{\lambda} d k \sin \theta_{m}\right)\right]+\omega(t)
\end{aligned}
$$

In (5), $\tilde{a}_{m}$ is the complex amplitude of the $m$-th target, $x_{m, \mathrm{r}, \mathrm{p}}(t)$ is the $p$-th respiration harmonic components of the $m$-th human, and $\zeta$ denotes the phase residual. Further, $\lambda$ is the wavelength of center frequency, $d$ is the distance between the adjacent antennae, $\theta_{m}$ is the angle of the $m$-th human, and $\omega(t)$ is the additive white Gaussian noise (AWGN) signal. In the $\mathrm{RX}$ component, the de-chirping method achieves the low complexity of the FMCW radar. In the de-chirping method, as the beat signal, the conjugation multiplication method of the FMCW TX signals $s_{\mathrm{lx}}^{*}(t)$ and the received FMCW signals $y_{k,},(t)$ are performed. The beat signal is represented such that

$$
p_{k, l}(t)=y_{k, l}(t) s_{\mathrm{tx}}^{*}(t) .
$$

The transformed FMCW signal $p_{k,}(t)$ is determined by a Nyquist sampling frequency $f_{S}=1 / T_{S}$ as the digital signal $p_{k, L}[n]$ where $a_{m}=\tilde{a}_{m} \exp \left(\omega_{s} \tau_{m}-\frac{\mu}{2} \tau_{m}{ }^{2}\right)$, and $q=1,2, \cdots, Q$. In the case $q=1$, the main respiration signal is yielded; in the case of $q$ $=2,3, \cdots, Q$ denotes the respiration harmonic components.

$$
\begin{gathered}
p_{k, l}[n]=\sum_{m=0}^{M-1}[a_{m} \underbrace{\exp \left(j 2 \pi \mu \tau_{m} T_{s} n\right)}_{\text {distance }} \underbrace{\exp \left(j\left(\frac{4 \pi}{\lambda} l T_{F}\left(x_{m, h}+\sum_{q=1}^{Q} x_{m, q, \mathrm{r}}\right)+\xi\right)\right)}_{\text {vital Doppler }} . \\
\underbrace{\exp \left(\frac{j 2 \pi}{\lambda} d k \sin \theta_{m}\right)}_{\text {angle }}]+\omega(t)
\end{gathered}
$$

In (7), the Nyquist sampling frequency is determined to be $f_{S}=1 / T_{S}$ and the digital data is represented in the vector $\boldsymbol{p}_{k, l}=\left[p_{k, l}[0], p_{k, l}[1], \ldots, p_{k, l}[N-1]\right]^{\mathrm{T}}$ in (7). $\boldsymbol{p}_{k, l}$ is expressed by the distance, the vital Doppler, and the angle terms, respectively, such that

$$
\boldsymbol{p}_{k, l}=\boldsymbol{\alpha} \boldsymbol{v}_{l} \boldsymbol{d}_{k} \boldsymbol{R}+\boldsymbol{\omega},
$$

where $\boldsymbol{\alpha}$ and $\boldsymbol{\omega}$ are the amplitude and noise vectors, respectively. In order to explain further, $\boldsymbol{a}=\left[a_{0}, a_{1}, \ldots, a_{N-1}\right]^{\mathrm{T}}$ and $\boldsymbol{\omega}=\left[\omega_{0}, \omega_{1}, \ldots, \omega_{N-1}\right]^{\mathrm{T}}$, the range vector is denoted by $\boldsymbol{R}$, i.e., $\boldsymbol{R}=\left[R\left(\tau_{0}\right), R\left(\tau_{1}\right), \ldots, R\left(\tau_{M}\right)\right]$, where $R\left(\tau_{m}\right)$ is the FMCW beat signal of delay $\tau_{m}$, i.e., $R\left(\tau_{m}\right)=\exp \left(j 2 \pi \mu \tau_{m} T_{s} n\right)$. The velocity vector $\boldsymbol{v}_{l}$ is shown such that

$$
\boldsymbol{v}_{l}=\operatorname{diag}\left[v_{l}(0), v_{l}(1), \ldots, v_{l}(M-1)\right],
$$

where $\operatorname{diag}(\cdot)$ is a matrix operator that the on-diagonal elements has all elements except zero and the off-diagonal elements are all zero. The $m$-th element of $\boldsymbol{v}_{l}$ is denoted by $v_{l}(m)$ and it is represented such that

$$
v_{l}(m)=\exp \left(j\left(\frac{4 \pi}{\lambda} l T_{F}\left(x_{m, h}+\sum_{q=1}^{Q} x_{m, q, \mathrm{r}}\right)+\xi\right)\right) .
$$

In (8), $\boldsymbol{d}_{k}$ is the angle vector, $\boldsymbol{d}_{k}=\operatorname{diag}\left[d_{k}(0), d_{k}(1), \ldots\right.$, $\left.d_{k}(M-1)\right]$, where $d_{k}(m)$ is shown such that

$$
d_{k}(m)=\exp \left(\frac{j 2 \pi}{\lambda} d k \sin \theta_{m}\right) .
$$

\section{PROPOSED SUPER-RESOLUTION DOA \\ ESTIMATION OF VITAL FMCW RADAR}

In this section, the super-resolution DOA estimation for vital FMCW radar with a wide array distance is proposed. This algorithm is aimed at enhancing the DOA resolution over that of the conventional estimator. Because their antenna arrays are not sufficiently large due to high cost and antenna distance $d=$ $\lambda / 2$, the angle resolution of conventional FMCW radar is low. Because this paper concentrates on angle detection, the Dop- 
pler information has been omitted.

\section{Distance Parameter Estimation by FFT}

Initially, the proposed algorithm finds the distance parameter of multiple targets using the received beat signal's FFT results [3]. After the 1D-FFT accomplishes the received signal, threshold detection is used to compute the distance index of various targets, and the FFT results of the distance index is acquired. The $1 \mathrm{D}-\mathrm{FFT}$ results $\boldsymbol{P}_{k, 1}=\left[P_{k, 1}[0], P_{k, 1}[1], \cdots, P_{k, 1}\right.$ $[N-1]]^{\mathrm{T}}$ at the 1 st chirp symbol-based distance information are represented such that

$$
\boldsymbol{P}_{k, 1}=\boldsymbol{W}_{N} \boldsymbol{p}_{k, 1},
$$

where $\boldsymbol{W}_{N}$ is the matrix of FFT, which consists of $N$ column vectors with $N \times 1$ elements, i.e., $\boldsymbol{W}_{N}=\left[W_{0}, W_{1}, \ldots, W_{N-1}\right]$. Further, the $u$-th column vector is represented by $W_{u}$ such that

$$
W_{u}=\left[1, \exp \left(-j \frac{2 \pi u}{N}\right), \ldots, \exp \left(-j \frac{2 \pi u(N-1)}{N}\right)\right]^{\mathrm{T}} .
$$

Of the FFT results, this paper can remove clutter using characteristics inherent to vital signals. The phase information of clutters does not change according to time; whereas, the phase of respiration and heartbeat have a change over time. This property was used to efficiently eliminate clutter. In order to obtain the distance of the human being, among the 1D-FFT's processing results that remove the clutters, the peak results $I=\left[I_{1}\right.$, $\left.I_{2}, \cdots, I_{M}\right]$ are obtained. When the distance $1 \mathrm{D}-\mathrm{FFT}$ 's peak value is utilized, the super-resolution DOA results with a wide array distance are accomplished in the next section.

\section{Proposed Method for the Angle Information}

Through the distance FFT's peak value for distance estimation, the angle detection with super-resolution can be accomplished. Prior to angle detection, we must consider the angular resolution and the total FOV when the array distance changes. In the FOV, the conventional angle range was set from $-90^{\circ}$ to $90^{\circ}$. This angle range is according to (11) where $d=\lambda / 2$ is swept from -1 to 1 . When the array distance $d$ is set to $\lambda$, the total FOV angle changes from $-30^{\circ}$ to $30^{\circ}$ while the angle range is swept from -1 to 1 using (11). Since conventional studies of vital radar consider a narrow FOV [5], this paper focuses on a narrow FOV. Under a narrow FOV, there is a margin to increase the spacing between arrays. Increasing the spacing between arrays can maintain resolution even if the number of arrays is reduced. By reducing the total number of arrays, the proposed scheme reduces system complexity while maintaining the same resolution.
In the case of angular resolution, we consider the ideal angular resolution $\Delta \theta$ such that [13]:

$$
\Delta \theta \approx \frac{0.886 \lambda}{K d \cos \theta} .
$$

In (14), in the case of an array distance of $d=\lambda / 2$, we obtain the ideal angular resolution. When the array distance $d$ is changed to $\lambda$, we achieve twice the improvement in resolution. In order to obtain the super-resolution angle results with $d=\lambda$, the proposed algorithm with a wide array distance and extrapolation method virtually generates data between antenna spacing to improve the resolution. The extrapolation-based DOA technique depends on modeling the extracted results as the output of a linear system of a rational system form, whereby

$$
H(z)=\frac{B(z)}{A(z)}=\frac{\sum_{k=0}^{h} b(k) z^{-k}}{1+\sum_{k=1}^{g} a(k) z^{-k}} .
$$

This mathematical model by the pole zero linear model in (15) is called an autoregressive-moving average (ARMA) model. This model is categorized as the ARMA model, AR model, and the moving-average (MA) model by the values of $g$ and $b$ in (15). Among these three linear models, the AR model with $h=$ 0 is the most widely used because the AR model results in very simple linear equations for the AR parameters. The typical method to estimate an AR parameter for estimating DOA information is the covariance method by [14]. Using extracted AR parameters, we can extrapolate the results of the extracted $i$ th target $\boldsymbol{P}_{K^{\prime}, i}^{\text {extra }}=\left[P_{1,1}\left[I_{i}\right], P_{2,1}\left[I_{i}\right], \ldots, P_{K, 1}\left[I_{i}\right], \ldots, P_{K^{\prime},[}\left[I_{i}\right]\right]^{\mathrm{T}}$ at the 1st chirp symbol where $K^{\prime}$ is the number of extrapolated signals. Finally, the extracted 1D-extrapolations are fed into the super-resolution algorithms. Of all the available super-resolution algorithms, this study selected the MUSIC algorithm. When the data extracted by only the distance index is processed by MUSIC $[15,16]$, the proposed algorithm obtains highresolution DOA results. When obtaining the DOA, the received signal's correlation matrix is needed to check a full rank. However, when the angular components of each target are similar to each other, that is, a coherent signal exists, the rank of the matrix can be reduced. The coherent signal refers to multiple targets in the same range cell. This coherent signal correlation matrix is meant as a drawback. This problem is solved by a smoothing method in the DOA direction [13]. The auto-correlation matrix $R_{X, i}$ of the extrapolation results of the extracted $i$-th target $\boldsymbol{P}_{U, i}^{\text {extra }}=\left[P_{m+0,1}\left[I_{i}\right], P_{m+1,1}\left[I_{i}\right], \ldots, P_{m+U-1,1}\left[I_{i}\right]\right]^{\mathrm{T}}$ at 1st chirp symbol that is $U \leq K^{\prime}$, is represented such that 


$$
\boldsymbol{R}_{X, i}=\sum_{m=0}^{K^{\prime}-U-1} \boldsymbol{P}_{U, i}^{\text {extra }}\left(\boldsymbol{P}_{U, i}^{\text {extra }}\right)^{\mathrm{H}}
$$

In order to reduce the correlation effect, the forwardbackward technique is represented such that

$$
\boldsymbol{R}_{f b, i}=\frac{1}{2}\left(\boldsymbol{R}_{X, i}+\boldsymbol{J} \boldsymbol{R}_{X, i}^{*} \boldsymbol{J}\right)
$$

where $J$ is the exchange matrix.

Through eigenvalue decomposition (EVD), the forwardbackward auto-correlation matrix $R_{f b, i}$ is performed [15] such that

$$
\boldsymbol{R}_{f b, i}=\left[\begin{array}{ll}
\boldsymbol{S} & \boldsymbol{N}
\end{array}\right]\left[\begin{array}{cccc}
\lambda_{0} & 0 & \vdots & 0 \\
0 & \lambda_{1} & \ddots & \vdots \\
\vdots & \ddots & \ddots & 0 \\
0 & \cdots & 0 & \lambda_{U-1}
\end{array}\right]\left[\begin{array}{c}
\boldsymbol{S}^{*} \\
\boldsymbol{N}^{*}
\end{array}\right]
$$

In (18), the signal eigenvector matrix $S=\left[s_{0}, \cdots, s_{P-1}\right]$ is matched with the signal subspace of the forward-backward auto-correlation matrix. The noise eigenvector matrix $N=\left[n_{0}, \cdots\right.$, $\left.n_{T-P-1}\right]$ represents the noise subspace of the auto-correlation matrix, and $\lambda_{n}$ denotes the $n$-th eigenvalues of $R_{f b, i}$. The total number of human beings at the same distance is denoted by $Q$. The largest $Q$ eigenvalues of $\lambda_{0}, \cdots, \lambda_{Q-1}$ are matched with the $Q$ signal eigenvectors of $S$. The other eigenvalues $\lambda_{Q}, \cdots, \lambda_{U-1}$ are matched with the noise eigenvectors of $N$ such that $\lambda_{Q}=\cdots=$ $\lambda_{U-1}=\sigma^{2}$. The eigenvalue is represented such that

$$
\lambda_{0} \geq \lambda_{1} \geq \cdots \geq \lambda_{Q-1} \geq \lambda_{Q}=\cdots \lambda_{U-1}=\sigma^{2} .
$$

The $P$ signal eigenvectors $S$ are the signal subspace and the $U$-Q-1 noise eigenvectors $N$ represent the noise subspace. The MUSIC algorithm has the characteristics that the steering DOA vector $a(\theta)$ and the noise eigenvectors $N$ are orthogonal such that

$$
a(\theta) N^{\mathrm{H}}=0,
$$

where $a(\theta)$ is defined as

$$
a(\theta)=\left[1, \exp \left(\frac{j 2 \pi}{\lambda} d \sin \theta\right), \ldots, \exp \left(\frac{j 2 \pi}{\lambda} d(K-1) \sin \theta\right)\right] .
$$

Using the property of (20), the angle MUSIC spectrum in the $i$-th target among $M$ humans can be estimated such that

$$
P_{\mathrm{MUSIC}, i}=\frac{1}{a(\theta) \boldsymbol{N}^{\mathrm{H}} \boldsymbol{N} a^{\mathrm{H}}(\theta)} .
$$

Thus, the proposed angle parameter estimator for vital FMCW radars is obtained.

\section{Summary of the Proposed Algorithm}

The major steps of the proposed algorithm in Fig. 2 are as follows:

- Step 1: The distance between multiple human beings is obtained using the FFT outputs of the received beat signal, i.e., the range bins are obtained.

- Step 2: Among FFT outputs, the clutters are removed using characteristics of the vital signals. The phase information of clutters does not change according to time, whereas the phase of respiration and heartbeat have a change over time. This property was used to efficiently eliminate clutter.

- Step 3: Among the distance FFT outputs in the which the clutters are removed, the maximum peaks among $M$ targets are obtained to effectively detect angle- that is, the range bins in which targets exist are selected among all the range bins.

- Step 4: Since the conventional studies of vital radar consider narrow a FOV [5], this paper also focused on narrow FOV. Under a narrow FOV, the margin to increase the spacing between arrays exists. After the array distance $d$ is set to $\lambda$, we obtain the total FOV from $-30^{\circ}$ to $30^{\circ}$ and achieve twice the improvement in DOA resolution.

- Step 5: The array data virtually increase using the extrapolation method.

- Step 6: By performing the MUSIC algorithm on the extrapolated array signal, improved DOA information is obtained.

\section{SIMULATIONS}

In this section, the estimation of the proposed angle algorithm is verified through various simulations and compared with the performance of conventional algorithms, such as the FFT and MUSIC.

\section{Simulation Environment}

In this section, two simulations are considered to verify the

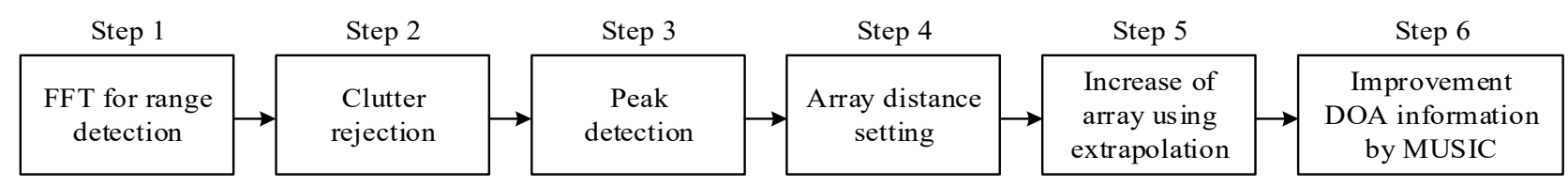

Fig. 2. Major steps in the proposed algorithm. 
Table 1. Summary of the simulation environment

\begin{tabular}{lc}
\hline \multicolumn{1}{c}{ Parameter } & Value \\
\hline Center frequency & $24 \mathrm{GHz}$ \\
Bandwidth & $1 \mathrm{GHz}$ \\
Chirp duration $(T)$ & $400 \mu \mathrm{s}$ \\
Guards period $\left(T_{i}\right)$ & $600 \mu \mathrm{s}$ \\
Number of samples per chirp $(N)$ & 2,000 \\
Number of chirp per one frame $(L)$ & 256 \\
\hline
\end{tabular}

estimation of the proposed DOA algorithm. Through the 1st simulation, the spectrum of the proposed algorithm, FFT, and MUSIC, for angle detection, are achieved. In the 2nd simulation, we can perform the algorithms' root-mean-square error (RMSE) according to the difference in angle and the same distance of the two targets. The SNR is defined by $\mathrm{SNR}=10 \log _{10}\left(\sigma_{s}^{2} / \sigma_{w}^{2}\right)$, where $\sigma_{s}^{2}$ denotes the power of the signal and $\sigma_{w}^{2}$ is the noise power. The simulation parameters of the FMCW radar are represented in Table 1 . The two targets are located at a distance of $3.0 \mathrm{~m}$ from the radar. The angle RMSE of each algorithm at a variety of SNR is performed $C$ times, where $C$ is the number of simulations. The RMSE is calculated by $\sqrt{\frac{1}{M C} \sum_{n=1}^{C} \sum_{m=1}^{M}\left(\hat{\theta}_{m, n}-\theta_{m, n}\right)^{2}}$ and $C$ is set to $10^{3}$, where $\theta_{m, n}$ and $\hat{\theta}_{m, n}$ are the actual DOA and the estimated DOA, respectively, of the $m$-th target at the $n$-th Monte Carlo simulation.

\section{Simulation Results}

Under these simulation environments, two targets are considered at the same distance from the radar and the interval distance between the two targets is close. The proposed scheme can distinguish between the two targets using the angle axis while conventional FFT and MUSIC cannot distinguish in terms of angle. In the case of $d=\lambda / 2$ and a number of arrays $K$ $=4$, the range of total FOV is from $-90^{\circ}$ to $90^{\circ}$ and the DOA resolution is about $25.4^{\circ}$ [14]. However, when the array distance $d$ is set to $\lambda$, we expected to obtain a range of the total FOV from $-30^{\circ}$ to $30^{\circ}$, and the DOA resolution to be about $12.7^{\circ}$ through simulation. The SNR parameter was configured to 20 dB. Fig. 3 represents the two targets' simulation environment. In Fig. 2, $R_{1}$ and $R_{2}$ are the ranges of the two targets, respectively. In this simulation, $R_{1}$ and $R_{2}$ are set to $3 \mathrm{~m}$ in order to confirm the angle resolution of each algorithm.

In Fig. 4, each algorithm's spectrum is simulated based on the angle interval at $K=4$. The proposed method sets the MUSICbased array at distance $=\lambda$ and $K=4$. In Fig. 4(a), the simulation results of MUSIC with $K=8$ and an array distance $=\lambda / 2$, MUSIC with $K=4$ and an array distance $=\lambda$, and the pro-

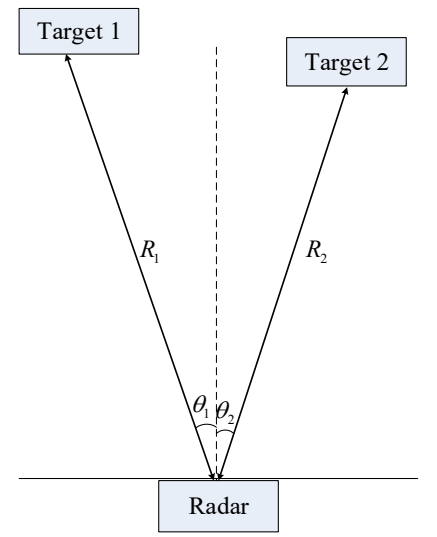

Fig. 3. The simulation environment of the two targets.

posed algorithm achieved two signal peaks in terms of the angle and they are represented with the reference data. When the two targets are located at $\theta=5^{\circ}$ and $\theta_{2}=25^{\circ}$, respectively, MUSIC with $K=8$, MUSIC with $K=4$, and the proposed algorithm can distinguish between the two targets appropriately. When the two targets are located very close together $\left(\theta=5^{\circ}\right.$ and $\theta_{2}=22^{\circ}$ ), the spectrum of MUSIC with $K=4$ in Fig. 4(b) has only a single peak in terms of the angle; whereas, the conventional MUSIC with $K=8$ and the proposed algorithm can still obtain the two peaks at $\theta=5^{\circ}$ and $\theta_{2}=22^{\circ}$ with the reference data. In Fig. 4(c), with $\theta=5^{\circ}$ and $\theta_{2}=20^{\circ}$, while conventional MUSIC with $K=4$ cannot separate the two targets adequately, the conventional MUSIC with $K=8$ and the proposed algorithm can obtain the two peaks compared with the reference data. From these results, we can conclude that the conventional MUSIC with $K=4$ cannot discern appropriately; however, the proposed algorithm can distinguish the two targets. Therefore, the proposed method is suitable as a super-resolution algorithm.

Fig. 5 represents the RMSEs of the results from the proposed and the conventional algorithms based on several angle separations for various SNR with $K=4$. We will concentrate on the RMSE results of the first target when the second target exists. The angle differences are set at $20^{\circ}, 17^{\circ}$, and $15^{\circ}$, respectively in Fig. 5. In Fig. 5(a), with an angle difference of $20^{\circ}$, the proposed and the conventional algorithms' RMSEs are similar. When the two persons' angle difference is closer to the resolution limitation, with an angle difference of $17^{\circ}$ as in Fig. 5(b), the proposed algorithm performs better than the MUSIC algorithm with $K=4$ under all SNRs. In Fig. 5(c), where the angle difference is $15^{\circ}$, the proposed algorithm and the MUSIC with $K=8$ have a low RMSE. The conventional MUSIC with $K=$ 4's RMSE performance is poor. Through these results, this section concludes that the proposed algorithm with $K=4$ 's estimation performance is similar to the conventional MUSIC with $K$ $=8$, while the proposed one has better estimation results than 


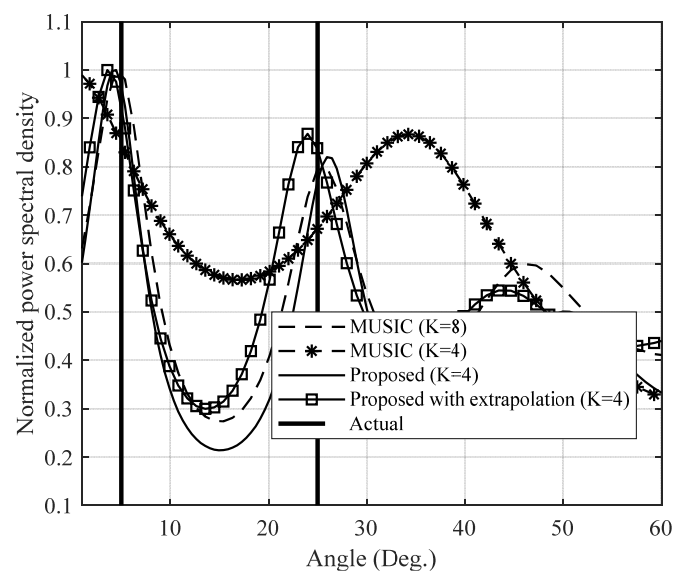

(a)

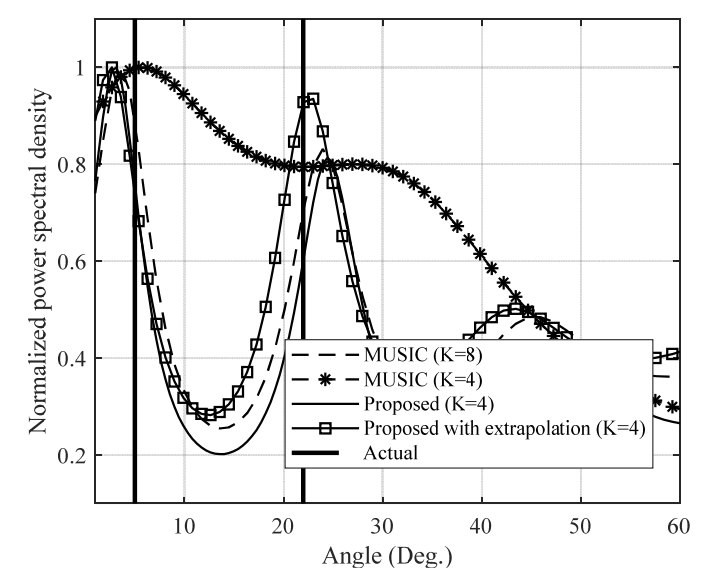

(b)

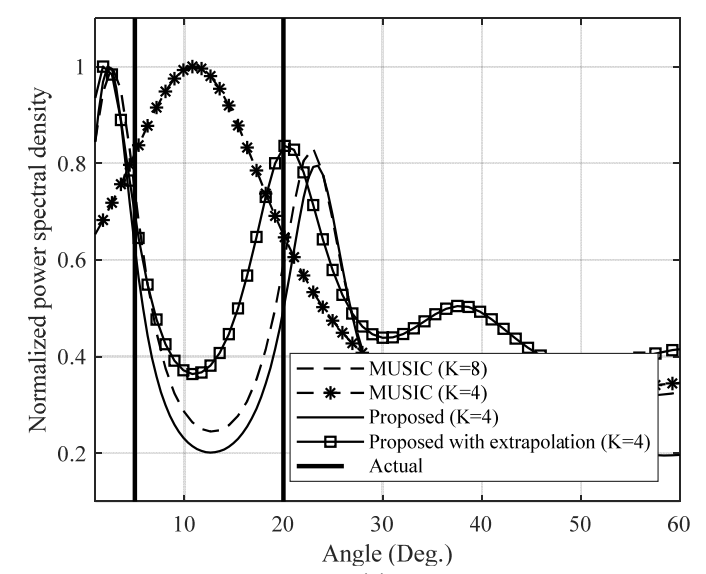

(c)

Fig. 4. Angle difference between the two targets at: (a) $20^{\circ}$, (b) $17^{\circ}$, and (c) $15^{\circ}$.

the MUSIC with $K=4$. According to Fig. 5, we set the size of the spectrum to 1,024 , thus we showed that angle estimation error reached about $1^{\circ}$.

\section{EXPERIMENTS}

This section presents the experimental results to verify the improvement by the proposed algorithm. The experiment was conducted in a chamber room. The total experimental parame-

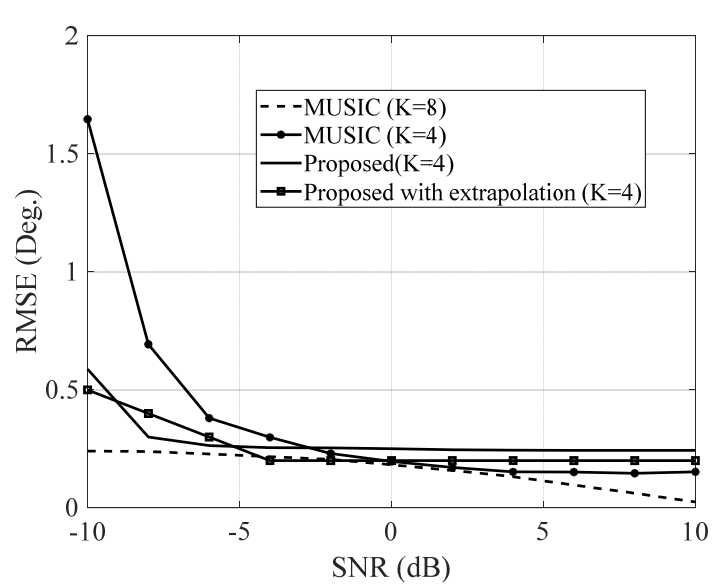

(a)

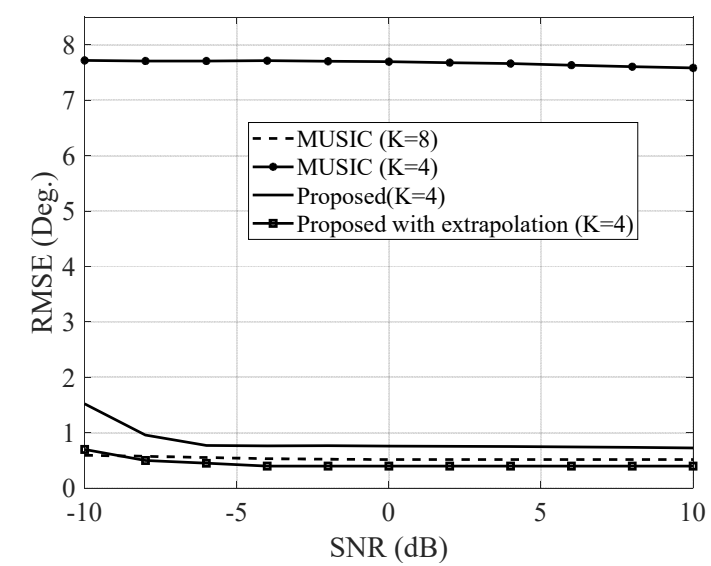

(b)

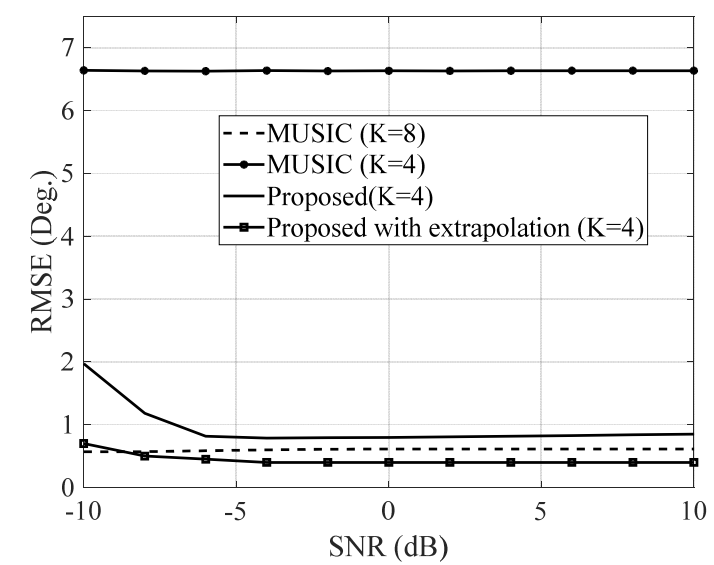

(c)

Fig. 5. RMSEs vs. SNR: (a) angle difference of $20^{\circ}$, (b) angle difference of $17^{\circ}$, and (c) the angle difference of $15^{\circ}$.

ters are represented in this section.

\section{Experimental Environments}

We utilized the $24 \mathrm{GHz}$ FMCW radar system with $2 \mathrm{TX}$ antennae and $8 \mathrm{RX}$ antennae in [13]. The TX block consists of a voltage-controlled oscillator (VCO), a phase-locked loop (PLL), and a frequency synthesizer. Finally, the VCO output is 
fed to the power amplifier and then the two TX antennae, respectively. In the RF system, the two TX antennae need not be deployed simultaneously. We chose only the TX antenna numbered 1 with a beam-width of $26^{\circ}$. No other antenna is used in this study. The RX assembly is composed of low noise amplifiers (LNAs), a variable gain amplifier (VGA), high-pass filters (HPFs), and low-pass filters (LPFs). Through the RX antennae and the LNAs, the reflected signals are received and are multiplied by the TX signals after which they pass the HPFs with $150 \mathrm{kHz}$ band-pass frequency. The HPF's purpose is to reject the DC-offset signal induced by the simple direct conversion architecture for the vital FMCW radar system [13]. After the HPFs, the beat signals of the eight channels were passed through the LPFs with $1.7 \mathrm{MHz}$ band-pass frequency. The noise figure of $\mathrm{RX}$ is $8.01 \mathrm{~dB}$ and the $\mathrm{RX}$ antenna gain is $10 \mathrm{~dB}$. The RX antenna azimuth beamwidth is $99.6^{\circ}$ and elevation beamwidth is $9.9^{\circ}$ [13].

We performed the experiment in the chamber whose details were explained in [13]. Two targets stood at the same distance from the radar in an anechoic chamber. The number of chirps per frame was set to 256 as depicted in Table 2. A 2048-point FFT was accomplished for the distance estimation.

\section{Experimental Results}

We assess the estimation performance of the proposed algorithm in this subsection. This paper focuses on detecting the DOA of the human. However, the signal part of the human with clutter removal in an indoor environment is similar to a stationary target in an anechoic chamber. After experimentation to remove the clutter in the indoor room, we were primed to analyze the DOA of the normal target in the chamber room.

In the case of two targets in indoor room, the proposed algorithm works at variable distances between the two humans shown in Fig. 6. The experimental results from the proposed algorithm was compared with the corresponding reference signal. In this part, the same parameters as in the simulation are required. In addition, experiments for the four cases completed are shown in Table 2. Two far-field targets, such as the humans in this experiment, were located at $R_{1}=2.9 \mathrm{~m}$ and $R_{2}=4.4,3.9$, 4.9 , and $5.4 \mathrm{~m}$, respectively. In terms of case I, II, III, and IV, when the two humans were located at certain distinct distances, the proposed result could classify the two humans from station-

Table 2. Summary of the simulation environment (unit: $\mathrm{m}$ )

\begin{tabular}{llcc}
\hline Parameter & $R_{1}$ & $R_{2}$ & $\left|R_{2}-R_{1}\right|$ \\
\hline Case I & 2.9 & 5.4 & 2.5 \\
Case II & 2.9 & 4.9 & 2.0 \\
Case III & 2.9 & 4.4 & 1.5 \\
Case IV & 2.9 & 3.9 & 1.0 \\
\hline
\end{tabular}

Real environment

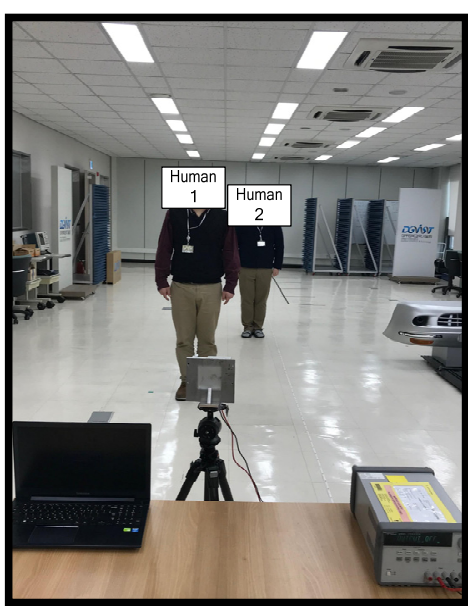

Fig. 6. Experimental environment with two humans.

ary clutter, simultaneously, as shown in Fig. 7. The proposed method effectively removes the clutter terms with a simple algorithm using the characteristics of vital signals. Cases I and II, which have a large distance, distinguished between the two humans from the conventional structure and the proposed structure. In the distance interval of case III, the conventional FFT algorithm did not distinguish the two humans, while the proposed structure could resolve them. Using the distances in case IV, the conventional FFT and the proposed algorithm did not distinguish two humans.

The experiment shows the DOA of the target is achieved through the distance estimation results from channel 1 to channel 8. Doppler estimation is not considered due to the two stationary targets; thus, the Doppler value of the two targets is zero. Through these experimental results, the results of the proposed structure can be represented as follows. The distance information of the two humans is obtained using the FFT outputs of the received beat signal, that is, the ranges are obtained in Fig. 8(a). Of the FFT outputs, the clutters are removed using the vital signals in Fig. 8(b). The two humans are located at $3 \mathrm{~m}$ from radar. From the distance FFT outputs of which the clutters are removed in Fig. 8(b), the maximum peaks are obtained in order to effectively detect the angle. Since the humans are in a narrow FOV, the array distance $d$ of the radar is set to $\lambda$. Finally, the data of the array virtually increases using extrapolation; then, the MUSIC algorithm performs on the extrapolated array signal to obtain the improved DOA information (Fig. 8(c)).

As shown in the detailed experimental results in Fig. 8, the experiment was conducted at distances of $2.5 \mathrm{~m}, 3.0 \mathrm{~m}$, and 3.5 $\mathrm{m}$. In order to obtain the angle, we utilized only the distance bins of $2.5 \mathrm{~m}, 3.0 \mathrm{~m}$, and $3.5 \mathrm{~m}$. In all experiments, the proposed algorithms can resolve the two targets, while the conventional MUSIC algorithm proved inferior. 


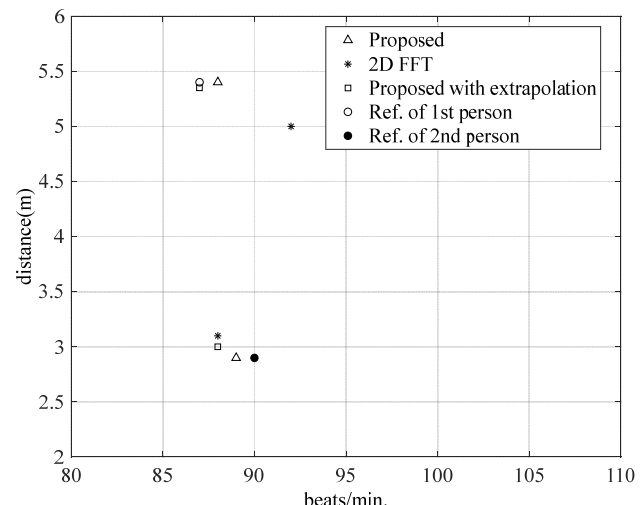

(a)

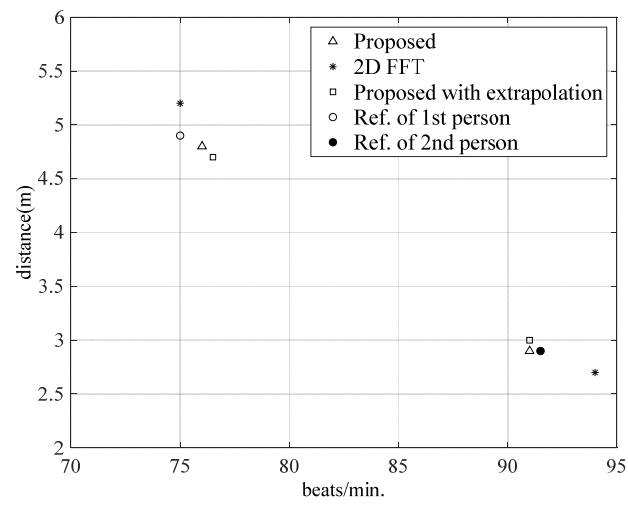

(b)

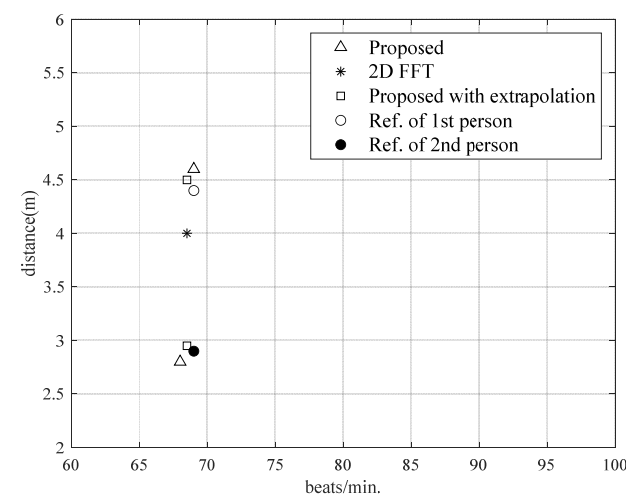

(c)

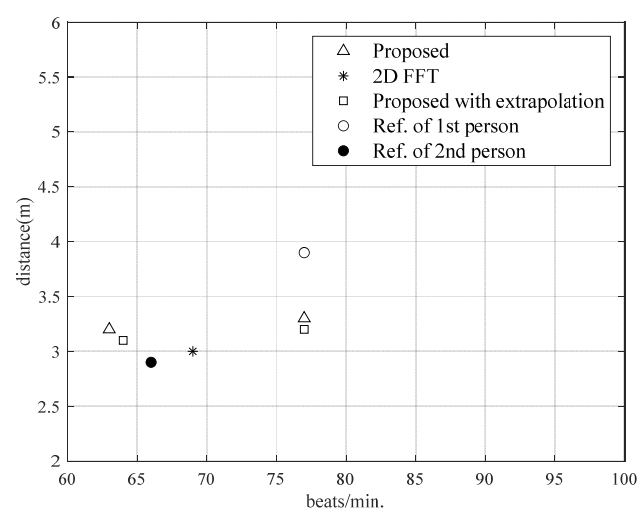

(d)

Fig. 7. Experimental results with clutter removal for (a) case I, (b) case II, (c) case III, and (d) case IV.

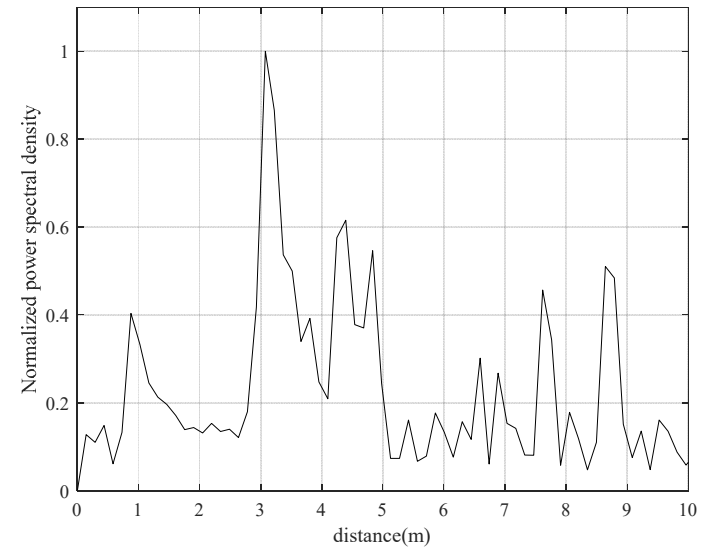

(a)

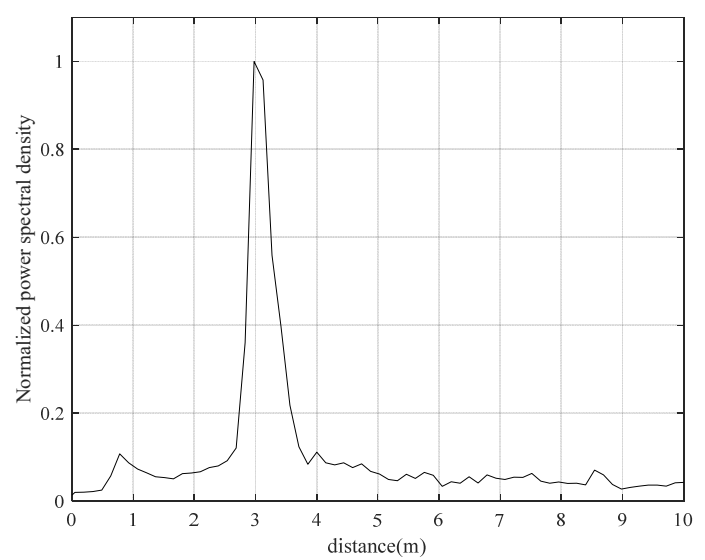

(b)

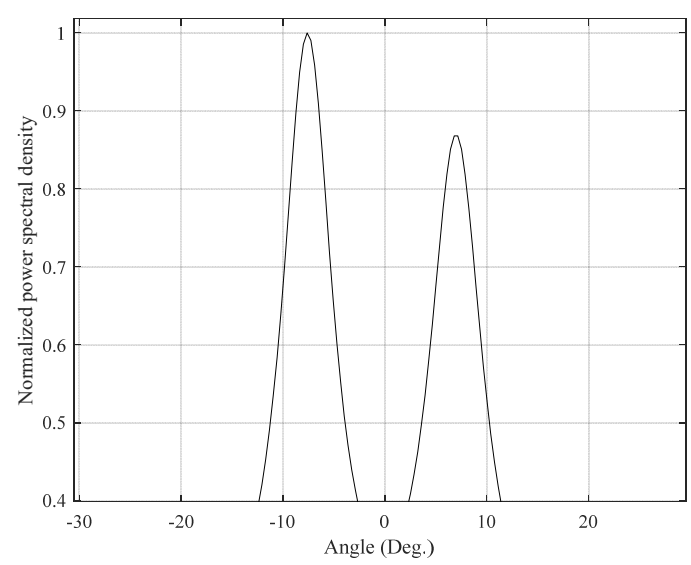

(c)

Fig. 8. The experimental results of the proposed algorithm: (a) range bins, (b) range bins with clutter removed, and (c) the DOA results.

Using the data from the distance bins at distance $=2.5 \mathrm{~m}$, Fig. 9(a) represents the experimental results with $K=4$ and 8 in the $\mathrm{RF}$ system represented in [13]. In Fig. 9(a), when $K=4$, the conventional MUSIC scheme cannot classify the two targets; whereas, when $K=8$, the proposed scheme and the conventional MUSIC can resolve the targets, even though the two targets are close. That is, the results of the proposed algorithm with $K=$ 4 is similar to the results obtained by the MUSIC algorithm 


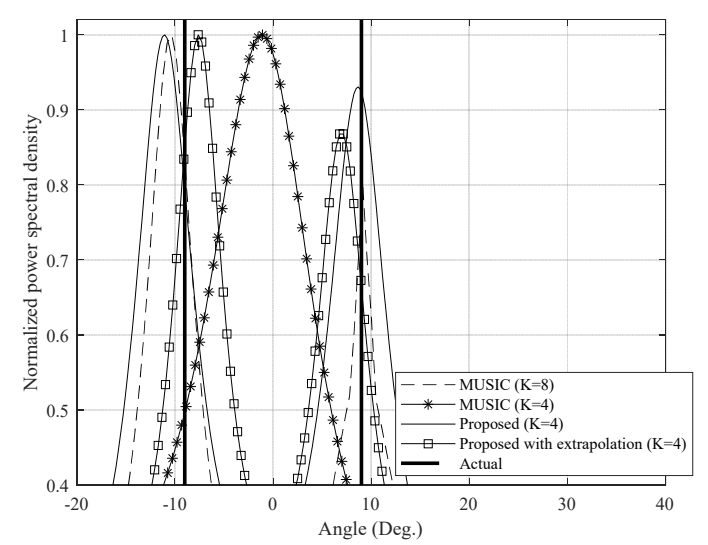

(a)

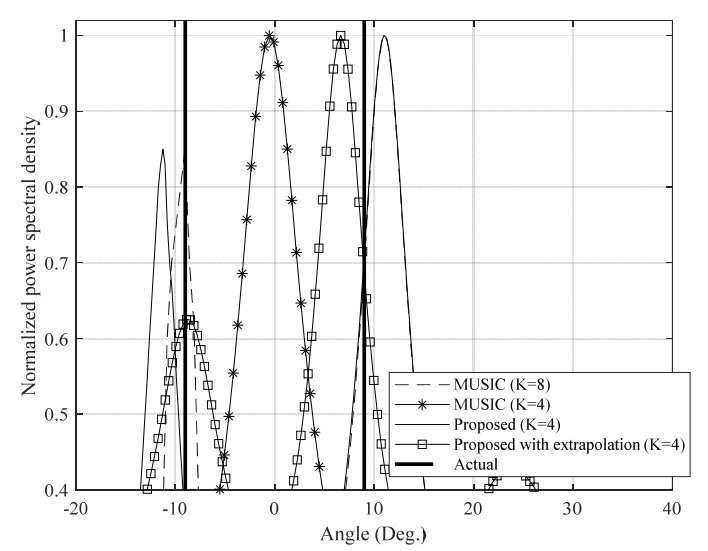

(b)

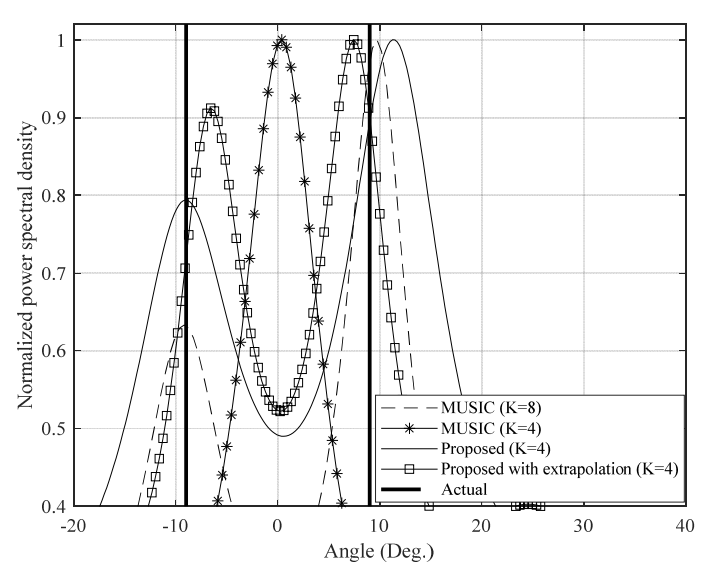

(c)

Fig. 9. Experimental DOA results at distance $=$ (a) $2.5 \mathrm{~m}$, (b) 3.0 $\mathrm{m}$, and (c) $3.5 \mathrm{~m}$.

with $K=8$. As the results of the experiment in Fig. 9(b) and (c), the angular resolution of the proposed structure shown in Fig. 9(a) is improved compared to the conventional structure. These results show that the proposed scheme achieves an improvement in the resolution due to the increased number of channels.

\section{CONCLUSION}

In this paper, a super-resolution-based DOA estimation strategy for vital FMCW radar with wide array distance and extrapolation was proposed. The proposed method considerably enhanced the angle estimation when two targets are very close to one another, compared with conventional algorithms where the angle estimate assumes that the distance between the antennae is not set to $\lambda / 2$ because the range of the FOV is not $\pm 90^{\circ}$. As the proposed algorithm uses $\lambda$ as the antenna distance, conventional algorithmic challenges, such as MUSIC's lowresolution problem, can be solved. The simulation results show that the proposed algorithm can correctly distinguish two targets when the angle difference between the two targets is $15^{\circ}$, whereas the MUSIC algorithm when $K=4$ cannot identify the same two targets as distinct. The RMSE of the proposed algorithm validates the results of the simulations. We analyzed experimentally that the proposed method can separate adjacent targets, while the MUSIC algorithm with $K=4$ cannot distinguish between them. Therefore, the proposed method is applicable to FMCW radar due to its high performance in parameter estimation. For further study, the MUSIC algorithm for antenna array processing needs band equalization due to channel mismatch. This issue will be the subject of future improvement of radar prototypes to enhance the milestone described herein.

This work was supported by DGIST and in part by the DGIST R\&D Program of the Ministry of Science, ICT and Future Planning, Korea (No. 21-IT-02).

\section{REFERENCES}

[1] T. Chen, B. Dai, D. Liu, J. Song, and Z. Liu, "Velodynebased curb detection up to 50 meters away," in Proceedings of 2015 IEEE Intelligent Vehicles Symposium (IV), Seoul, South Korea, 2015, pp. 241-248.

[2] M. Tan, B. Wang, Z. Wu, J. Wang, and G. Pan, "Weakly supervised metric learning for traffic sign recognition in a LIDAR-equipped vehicle," IEEE Transactions on Intelligent Transportation Systems, vol. 17, no. 5, pp. 1415-1427, 2016.

[3] S. Kim, D. Oh, and J. Lee, "Joint DFT-ESPRIT estimation for TOA and DOA in vehicle FMCW radars," IEEE $A n^{-}$ tennas and Wireless Propagation Letters, vol. 14, pp. 17101713, 2015.

[4] M. S. Lee, V. Katkovnik, and Y. H. Kim, "System modeling and signal processing for a switch antenna array radar," IEEE Transactions on Signal Processing, vol. 52, no. 6, pp. 1513-1523, 2004.

[5] S. Kim and K. K. Lee, "Low-complexity joint extrapolationMUSIC-based 2-D parameter estimator for vital FMCW radar," IEEE Sensors Journal, vol. 19, no. 6, pp. 2205-2216, 2019. 
[6] S. Saponara and B. Neri, "Radar sensor signal acquisition and multidimensional FFT processing for surveillance applications in transport systems," IEEE Transactions on Instrumentation and Measurement, vol. 66, no. 4, pp. 604-615, 2017.

[7] F. Wan, W. P. Zhu, and M. N. S. Swamy, "Spacial extrapolation-based blind DOA estimation approach for closely spaced sources," IEEE Transactions on Aerospace and Electronic systems, vol. 46, no. 2, pp. 569-582, 2010.

[8] V. K. Nguyen, M. D. Turley, and G. A. Fabrizio, "A new data extrapolation approach based on spectral partitioning," IEEE Signal Processing Letters, vol. 23, no. 4, pp. 454-458, 2016.

[9] Z. Peng, J. M. Munoz-Ferreras, Y. Tang, C. Liu, R. GomezGarcia, L. Ran, and C. Li, "A portable FMCW interferometry radar with programmable low-IF architecture for 1ocalization, ISAR imaging, and vital sign tracking," IEEE Transactions on Microwave Theory and Techniques, vol. 65, no. 4, pp. 1334-1344, 2017.

[10] Z. Peng, L. Ran, and C. Li, "A K-band portable FMCW radar with beamforming array for short-range localization and vital-Doppler targets discrimination," IEEE Transactions on Microwave Theory and Techniques, vol. 65, no. 9, pp. 3443-3452, 2017.

[11] M. Nosrati, S. Shahsavari, S. Lee, H. Wang, and N. Tavassolian, "A concurrent dual-beam phased-array Doppler

Sangdong Kim

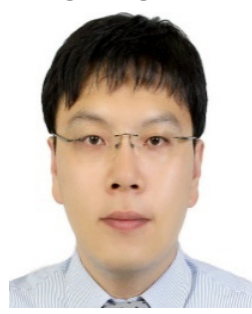

was born in Seoul, South Korea in 1981. He received his B.S. degree in Electronics Engineering from Hanyang University, Ansan, in 2004, his M.S. degree in Electronics Engineering from Hanyang University, Seoul, in 2006 and his Ph.D. degree in Electronics Engineering from Kyungpook National University, Daegu in 2018. From 2015 to 2016, he was a visiting scholar at the University of Florida. From 2006, he has been working as a Senior Researcher with the Division of Automotive Technology, the Daegu Gyeongbuk Institute of Science \& Technology (DGIST), Daegu, South Korea. And, from 2020, he has been working as an Assistant professor with the Department of Interdisciplinary Engineering(Adj.), the DGIST. His research interests include superresolution algorithms, automotive radar, and vital radar. radar using MIMO beamforming techniques for shortrange vital-signs monitoring," IEEE Transactions on Antennas and Propagation, vol. 67, no. 4, pp. 2390-2404, 2019.

[12] Y. Huang, Y. Zhang, W. Li, Y. Zhang, and Z. Wang, "Angular superresolution for scanning radar with music based on virtual array mapping," in Proceedings of 2015 IEEE Radar Conference (RadarCon), Arlington, VA, 2015, pp. 03240328.

[13] B. Kim, S. Kim, and J. Lee, "A novel DFT-based DOA estimation by a virtual array extension using simple multiplications for FMCW radar," Sensors, vol. 18, no. 5, pp. 1560-1576, 2018.

[14] B. W. Choi, E. H. Bae, J. S. Kim, and K. K. Lee, "Improved prewhitening method for linear frequency modulation reverberation using dechirping transformation," The Journal of the Acoustical Society of America, vol. 123, no. 3, pp. EL21EL25, 2008.

[15] F. Belfiori, W. van Rossum, and P. Hoogeboom, "Coherent MUSIC technique for range/angle information retrieval: application to a frequency-modulated continuous wave MIMO radar," IET Radar, Sonar \& Navigation, vol. 8, no. 2,pp. 75-83, 2014.

[16] S. Kim and K. Lee, "A low complexity based spectrum partitioning-ESPRIT for noncontact vital radar," Elektronika ir Elektrotechnika, vol. 23, no. 2, pp. 54-58, 2017.

Bongseok Kim

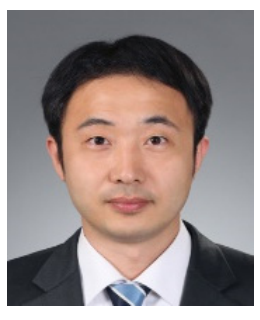

received his B.S. degree in Electronics Engineering in 2006 and M.S. and Ph.D. degrees in Information and Communications Engineering from Yeungnam University, South Korea, in 2009 and 2014, respectively. Since 2014, he has been working at the Daegu Gyeongbuk Institute of Science \& Technology (DGIST), South Korea, as a senior research engineer. His current interests include multi-functional radar systems and radar signal processing. 


\section{Youngseok Jin}

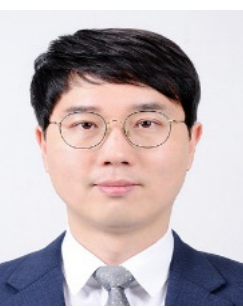

received his B.S. and M.S. degrees in Communication Engineering from Daegu University, South Korea in 2010 and 2012, respectively. Since 2012, he has been working at the Daegu Gyeongbuk Institute of Science \& Technology (DGIST), Korea, as a researcher. His current interests include radar signal processing and implementation in FPGA/DSP.
Jonghun Lee

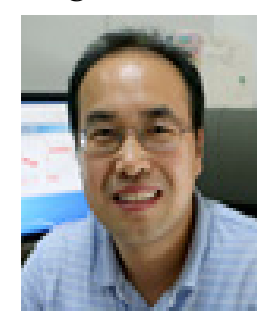

received B.S. degree in Electronics Engineering from SungKyunKwan University, South Korea, in 1996 and his M.S. and Ph.D. degrees in Electrical and Electronics and Computer Science from SungKyunKwan University in 1998 and 2002, respectively. From 2002 to 2005, he worked at Samsung Electronics Company as a Senior Research engineer. Since 2005, he has been working in the Division of $\mathrm{Au}-$ tomotive Technology, the Daegu Gyeongbuk Institute of Science \& Technology (DGIST), South Korea, as a principal research engineer. And, from 2020, he has been working as a Professor with the Department of Interdisciplinary Engineering(Adj.) of the DGIST. He is an IEEE senior member. His primary research interests are detection, tracking, recognition for radar (FMCW \& UWB radar), radar-based vehicle sensing and radar signal processing, and sensor fusion. 